\title{
REMARKS ON THE STACK OF COHERENT ALGEBRAS
}

\author{
MAX LIEBLICH
}

\begin{abstract}
We consider the stack of coherent algebras with proper support, a moduli problem generalizing Alexeev and Knutson's stack of branchvarieties to the case of an Artin stack. The main results are proofs of the existence of Quot and Hom spaces in greater generality than is currently known and several applications to Alexeev and Knutson's original construction: a proof that the stack of branchvarieties is always algebraic, that limits of one-dimensional families always exist, and that the connected components of the stack of branchvarieties are proper over the base under certain hypotheses on the ambient stack.
\end{abstract}

\section{INTRODUCTION}

In a recent paper [1], Alexeev and Knutson consider a moduli problem closely related to the Hilbert scheme: the stack of branchvarieties. They focus on branchvarieties over a projective scheme, and they prove (among other things) that the stack has proper components. The question of the existence of limits along discrete valuation rings is left open for not necessarily projective schemes, or, more generally, for algebraic spaces, as is the question of quasi-compactness of the connected components of the stack.

A branchvariety over $Y$ is, in particular, a finite morphism. Abandoning the projective methods of [1], we give an approach to the stack of branchvarieties using standard abstract methods applied to the stack of all finite morphisms with proper support. In particular, we give an abstract "construction" of (a mild generalization of) Alexeev and Knutson's stack of branchvarieties over any Deligne-Mumford stack. Then we show that the stack satisfies the valuative criterion of properness in full generality. When the stack has quasi-projective coarse moduli space, we show that the connected components of the stack of branchvarieties are proper. In fact, one can give (somewhat contrived) explicit numerical constraints which ensure boundedness of a collection of branchvarieties, if desired.

Among other things, our methods also yield proofs of the algebraicity of the usual stacks under very general conditions: Quot spaces for quasi-coherent sheaves of finite presentation with proper support on an Artin stack of finite presentation and (as pointed out to us by Starr) Hom spaces from a proper Artin stack of finite presentation to a global quotient by a flat linear algebraic group scheme. This gives generalizations of various recent results of Starr and Olsson contained in [10], [12], and [14]. In fact, 2.3.4 gives a natural complement to Theorem 1.1 of [10] for target stacks with potentially positive-dimensional stabilizers (but which are required to be global quotients). These applications are taken up in $\S 2$ and its subsections.

As the initial motivation for this work was to understand Alexeev and Knutson's stack, we use the rest of this introduction to sketch the main results concerning branchvarieties. Throughout, $Y \rightarrow S$ is a Deligne-Mumford stack of finite presentation over an excellent algebraic space. Somewhat more general results are 
obtainable in certain cases (as in the recent work of Starr [14]), but we restrict ourselves to this case because it is easier to describe and has some potentially interesting applications.

Definition 1.1. A family of branchvarieties over $Y$ parametrized by an affine scheme $T \rightarrow S$ is a proper flat Deligne-Mumford stack of finite presentation $X \rightarrow T$ with geometrically reduced fibers equipped with a finite $T$-morphism $X \rightarrow Y_{T}$.

Note that when $Y$ has automorphisms, we allow $X$ to have automorphisms. The locus consisting of branchvarieties with $X$ an algebraic space defines an open substack, but properness requires that we allow $X$ to acquire automorphisms in the limit. (Indeed, one can easily make examples where the presence of automorphisms is necessary in the limit by considering the Hilbert scheme of a weighted projective stack.) Of course, when $Y$ is an algebraic space (resp. scheme), $X$ will also be an algebraic space (resp. scheme).

We will prove the following.

Theorem 1.2. Branchvarieties over $Y$ form an Artin stack $\mathfrak{B} r_{Y / S}$ locally of finite presentation over $S$.

The most natural way to build the stack is as a stack of flat families of algebras over the structure sheaf of $Y$, something we sketch in section 2 .

Theorem 1.3. Let $T \rightarrow S$ be a discrete valuation scheme with generic point $\eta$. Suppose $X_{\eta} \rightarrow Y_{\eta}$ is a branchvariety. There is a finite totally ramified extension $T^{\prime} / T$ and a family of branchvarieties $X \rightarrow Y_{T^{\prime}}$ extending the generic family. Given two such families $X_{1}$ and $X_{2}$ and an isomorphism $\varphi:\left(X_{1}\right)_{\eta} \rightarrow\left(X_{2}\right)_{\eta}$ over $Y_{\eta}$, there is a unique isomorphism $X_{1} \rightarrow X_{2}$ over $Y$ extending $\varphi$.

The key to proving 1.3 is to note that both parts - the existence and uniqueness are local in the étale topology. Uniqueness then allows us to glue the local solutions to get a global limit.

Theorem 1.4. If $S$ is Noetherian and either 1) $Y$ admits a proper flat cover by a quasi-projective algebraic space over $S$, or 2) $Y$ is a quotient stack whose coarse moduli space is quasi-projective over $S$, then $\mathfrak{B} r_{Y / S}$ is a disjoint union of proper S-stacks.

Having proven that limits exist, the point is to prove that the connected components are quasi-compact. This is proved using the various numerical invariants provided by polarizations of coverings or of the coarse moduli space.

\section{ACKNOWLEDGMENTS}

I would like to thank Jason Starr and the referee for many helpful comments and suggestions, and in particular for strongly suggesting inclusion of sections 2.2 and 2.3 .

\section{THE STACK OF COHERENT ALGEBRAS WITH PROPER SUPPORT}

In this section, we sketch one approach to studying the stack of finite morphisms (and ultimately proving 1.2). The recent preprint of Starr [14] provides a general method for considering a whole slew of relatively algebraic morphisms of stacks. However, as Starr has pointed out to us, in the case of branchvarieties his method 
will only produce the open substack of our construction consisting of branchvarieties which are algebraic spaces. It is thus with the goal of producing a full compactification of the moduli problem that we present the following method.

2.1. Algebraicity of the stack. In this section, we show that the collection of finite morphisms with proper support to an Artin stack of finite presentation over an excellent algebraic space is itself an Artin stack locally of finite presentation over the base.

Theorem 2.1.1. Let $Y \rightarrow S$ be an Artin stack of finite presentation over an excellent algebraic space. The stack $\mathrm{Coh}_{Y / S}$ of flat families of finitely presented quasi-coherent sheaves on $Y$ with proper support over $S$ is an Artin stack locally of finite presentation over $S$.

Proof. This is a straightforward application of Artin's theorem, using the stacky Grothendieck existence theorem proven by Olsson in [11] and the deformation theory of Illusie for modules in a topos. When $Y$ is a Deligne-Mumford stack, one can simply use the étale topos of $Y$. However, when $Y$ is an arbitrary Artin stack, one must work with Cartesian modules on a simplicial topos generated by a smooth cover of $Y$. (To apply the theory of Illusie one must in addition note that any infinitesimal deformation of a Cartesian module is Cartesian; this ensures that the full Ext groups parametrize obstructions and deformations.) The reader unfamiliar with these methods is referred to [9] for further details (but should note that deformation theory of sheaves is not explicitly developed there). The uncomfortable reader may choose to only think about Deligne-Mumford stacks.

Remark 2.1.2. This gives a generalization of Théorème 4.6.2.1 of [8], which only handles the case of projective morphisms $Y \rightarrow S$ of Noetherian schemes.

Proposition 2.1.3. Let $Y \rightarrow S$ be a morphism of finite presentation between Artin stacks. Let $\mathscr{F}$ and $\mathscr{G}$ be finitely presented quasi-coherent sheaves on $Y$ such that $\mathscr{G}$ is $S$-flat and the support of $\mathscr{G}$ is proper over $S$. The stack of homomorphisms $\underline{\operatorname{Hom}}(\mathscr{F}, \mathscr{G}) \rightarrow S$ is representable by algebraic spaces locally of finite type.

Proof. The stack $\underline{\operatorname{Hom}}(\mathscr{F}, \mathscr{G})$ is the stack on the big fppf site of $S$ whose fiber category over a 1-morphism from a scheme $W \rightarrow S$ is the set of homomorphisms $\operatorname{Hom}\left(\mathscr{F}_{W}, \mathscr{G}_{W}\right)$. It is easy to check that this is a sheaf on the big fppf topos of $S$; to show that it is a relative algebraic space it suffices to prove this after pulling back to a smooth cover of $S$, so we may assume that $S$ is a scheme. Now we can apply Artin's theorem (for algebraic spaces!), but using elementary arguments in place of Illusie's theory. E.g., given a homomorphism from $\mathscr{F}_{W} \rightarrow \mathscr{G}_{W}$ and an infinitesimal extension of $W$ by an ideal $I$, basic homological algebra yields an obstruction to lifting the homomorphism in $\operatorname{Ext}^{1}(\mathscr{F}, I \otimes \mathscr{G})$. The set of lifts is a torsor under $\operatorname{Hom}(\mathscr{F}, I \otimes \mathscr{G})$ (again by basic algebra). The $S$-flatness of $\mathscr{G}$ is used in verifying the Schlessinger conditions on the functor $\underline{\operatorname{Hom}}(\mathscr{F}, \mathscr{G})$. The algebraic approximation of formal deformations is accomplished using the Grothendieck Existence Theorem for Artin stacks proven by Olsson [11]. The rest is completely straightforward.

Remark 2.1.4. One easily deduces from Corollaire 7.7 .8 of [4] that 2.1.3 holds when $Y \rightarrow S$ is a projective morphism of locally Noetherian schemes, and moreover that $\underline{\operatorname{Hom}}(\mathscr{F}, \mathscr{G})$ is representable locally on $S$ by the kernel of a linear homomorphism of geometric vector bundles. The method above already gives a generalization when 
$Y \rightarrow S$ is a proper morphism of finite presentation between schemes, something which seems difficult to prove with the techniques of [4], except when $\mathscr{F}$ is the cokernel of a homomorphism between locally free sheaves of finite rank.

Proposition 2.1.5. Let $Y \rightarrow S$ be a morphism of finite presentation of Artin stacks and $\mathscr{F}$ an $S$-flat finitely presented quasi-coherent sheaf on $Y$ with proper support. The stack of commutative algebra structures on $\mathscr{F}$ is represented by a relative algebraic space of finite presentation over $S$.

Proof. To give an algebra structure on $\mathscr{F}$ is to give 1 ) a map $\mu: \mathscr{F} \otimes \mathscr{F} \rightarrow \mathscr{F}$ and 2) a map $v: \mathscr{O} \rightarrow \mathscr{F}$ such that $\mu$ defines a commutative and associative pairing and $v$ defines a unit for this pairing. The conditions of commutativity, associativity, and unit define a closed substack of the stack of pairs $\underline{\operatorname{Hom}}(\mathscr{F} \otimes \mathscr{F}, \mathscr{F}) \times \underline{\operatorname{Hom}}(\mathscr{O}, \mathscr{F})$. Since $\mathscr{F}$ is $S$-flat, the latter stack is algebraic. The result follows.

2.2. Quot spaces. Using the above methods, one can prove that algebraic Quot spaces exist on Artin stacks, generalizing the main result of [12].

2.2.1. Let $Y \rightarrow S$ be a morphism of finite presentation between Artin stacks and $\mathscr{F}$ and $\mathscr{G}$ quasi-coherent sheaves of finite presentation on $Y$ such that $\mathscr{G}$ is $S$ flat and has proper support. We may apply 2.1.3 to produce an Artin stack with a representable morphism $\underline{\operatorname{Hom}}(\mathscr{F}, \mathscr{G}) \rightarrow S$ locally of finite presentation which parametrizes homomorphisms $\mathscr{F} \rightarrow \mathscr{G}$.

Lemma 2.2.2. There is an open substack of $\underline{\operatorname{Hom}}(\mathscr{F}, \mathscr{G})$ parametrizing surjective homomorphisms.

Proof. By Nakayama's lemma (and the faithful flatness of field extensions), given a scheme $W \rightarrow S$, a homomorphism $\varphi: \mathscr{F}_{W} \rightarrow \mathscr{G}_{W}$ is surjective if and only if it is surjective when pulled back to all geometric points of $W$. Thus, to show that surjections are represented by an open substack, it suffices to show that when $W$ is reduced the locus of points in $W$ over which $\varphi$ is surjective is open. Since the formation of $\operatorname{coker} \varphi$ commutes with base change, this is the same as showing that given a quasi-coherent sheaf $\mathscr{K}$ on $Y_{W}$ of finite presentation whose support is proper over $W$, the locus of points $w \in W$ such that $\mathscr{K}_{w}=0$ is open. By Nakayama's lemma, the set of such $t$ is precisely the complement of the image of the support of $\mathscr{K}$. Since the support is proper, it has closed image.

2.2.3. Let $Y \rightarrow S$ be a morphism of finite presentation between Artin stacks and $\mathscr{F}$ a quasi-coherent sheaf of $\mathscr{O}_{Y}$-modules of finite presentation. We can define a presheaf on the category of $S$-schemes as follows: For any $S$-scheme $W \rightarrow S$, let $\operatorname{Quot}_{Y / S}(\mathscr{F})(W)$ be isomorphism classes of surjective homomorphisms $\mathscr{F}_{W} \rightarrow \mathscr{G}$ with $\mathscr{G}$ a $W$-flat quasi-coherent sheaf on $Y_{W}$ of finite presentation with support proper over $W$. Since sheaves and homomorphisms glue in the fpqc topology on an Artin stack, it is easy to see that $\operatorname{Quot}_{Y / S}(\mathscr{F})$ is a sheaf on the big fpqc topos on $S$. In the parlance of $\S 14$ of [8] (slightly generalized to base stacks rather than base schemes), the formation of $\operatorname{Quot}_{Y / S}(\mathscr{F})$ is a "construction locale."

Proposition 2.2.4. There is a representable morphism locally of finite presentation Quot $_{Y / S}(\mathscr{F}) \rightarrow S$ whose associated sheaf on the big fpqc site of $S$ is isomorphic to $\operatorname{Quot}_{Y / S}(\mathscr{F})$. 
Proof. By standard limiting methods, it suffices to prove the result when $S$ is an excellent algebraic space. Let $\mathscr{G}$ be the universal sheaf on $Y \times \operatorname{Coh}_{Y / S}$ and consider the $\operatorname{Coh}_{Y / S}$-stack $\underline{\operatorname{Hom}}_{Y \times S} \operatorname{Coh}_{Y / S}\left(\mathscr{F}_{\mathrm{Coh}_{Y / S}}, \mathscr{G}\right)$, which is an Artin stack locally of finite presentation by 2.1.1 and 2.1.3. By 2.2.2 there is an open substack parametrizing surjective morphisms, and this is precisely the stack $\underline{\text { Quot }}_{Y / S}(\mathscr{F})$.

2.3. Hom stacks. Jason Starr has pointed out to us that we can refine the results of $\S 2.1$ in another direction to study $\operatorname{Hom}$-stacks $\operatorname{Hom}(X, Y)$ where $X$ is a proper Artin stack and $Y$ is a suitable quotient stack.

Let $S$ be an excellent algebraic space and $Y \rightarrow S$ a proper Artin stack of finite presentation.

Lemma 2.3.1. There is an open substack $\mathrm{LF}_{Y / S} \subset \mathrm{Coh}_{Y / S}$ parametrizing locally free sheaves of finite rank.

Proof. This follows immediately from Nakayama's lemma and reduction to the Noetherian case.

Given an Artin $S$-stack $X \rightarrow S$, one can define a Hom-stack $\operatorname{Hom}_{S}(Y, X)$ to have objects over $W \rightarrow S$ the groupoid of 1-morphisms $Y_{W} \rightarrow X_{W}$. Choosing a flat presentation $V \rightarrow Y$, the objects of $\operatorname{Hom}_{S}(Y, X)$ over $W$ are the same thing as simplicial objects of $X_{W}$ over the simplicial scheme coming from the collection fiber products $V_{W} \times_{Y_{W}} \cdots \times_{Y_{W}} V_{W}$. (In other words, passing to the associated simplicial object defines a natural equivalence of groupoids.) From this point of view, it is clear that $\operatorname{Hom}_{S}(Y, X)$ is an fppf $S$-stack.

One such stack we can take for $X$ is $\mathrm{BGL}_{n}$, the classifying stack of $\mathrm{GL}_{n}$-torsors.

Lemma 2.3.2. The stack $\operatorname{Hom}_{S}\left(Y, \mathrm{BGL}_{n}\right)$ is an Artin stack locally of finite presentation over $S$.

Proof. It is easy to check that giving an object of $\operatorname{Hom}_{S}\left(Y, \mathrm{BGL}_{n}\right)$ over $W \rightarrow S$ is the same as giving the $\mathrm{GL}_{n}$-torsor on $Y_{W}$ associated to a locally free sheaf of $\mathscr{O}_{Y_{W}}$-modules of rank $n$. Applying 2.3.1 yields the result.

Lemma 2.3.3. Let $X \rightarrow S$ and $Y \rightarrow S$ be Artin stacks locally of finite presentation over $S$ with $Y$ proper and $X$ separated and let $\varphi: X \rightarrow Y$ be a representable $S$ morphism. There is an Artin stack $\Sigma(\varphi)$ locally of finite presentation over $S$ whose objects over $W \rightarrow S$ are pairs $(\psi, \gamma)$ with $\psi: Y_{W} \rightarrow X_{W} a W$-morphism and $\gamma: \varphi \psi \stackrel{\sim}{\rightarrow}$ id an isomorphism.

Proof. First, note that since $X \rightarrow Y$ is representable, it is easy to see that the stack $\Sigma(\varphi)$ is the stack associated to a sheaf on $S$.

We may assume that $S$ is Noetherian. Consider the Quot space $\operatorname{Quot}_{X / S}\left(\mathscr{O}_{X}\right)$, whose points correspond to flat families of closed substacks of $X$. The universal family $\mathcal{Z} \subset X \times_{S}$ Quot $_{X / S}(\mathscr{O})$ and the map $X \rightarrow Y$ give rise to a representable

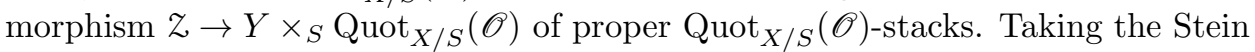
factorization yields a finite coherent $\mathscr{O}_{Y_{\text {Quot }}}$-algebra $\mathscr{A}$. The locus of $Y_{\text {Quot }}$ over which the natural map $\mathscr{O}_{Y_{\text {Quot }}} \rightarrow \mathscr{A}$ is an isomorphism is an open substack of $Y_{\text {Quot }}$. The complement of its image in $\operatorname{Quot}_{X / S}$ yields an open substack $\mathscr{U} \subset \operatorname{Quot}_{X / S}(\mathscr{O})$ over which the map $z \rightarrow Y_{\mathrm{Quot}_{X / S}(\mathscr{O})}$ is an isomorphism. It is immediate that $\Sigma(\varphi)$ is represented by $\mathscr{U}$. 
With this in hand, we can prove algebraicity of Hom-stacks to global quotients by linear algebraic group schemes.

Proposition 2.3.4. Let $X=[Z / G]$ be a quotient stack with $Z$ separated and of finite presentation over $S$ and $G$ an $S$-flat linear algebraic group scheme. The stack $\operatorname{Hom}_{S}(Y, X)$ is an Artin stack locally of finite presentation over $S$.

Proof. Since algebraicity is étale-local, we may assume that $S$ is an excellent quasicompact scheme. There is an inclusion $G \hookrightarrow \mathrm{GL}_{n}$ for some $n$. Since $G$ is $S$-flat, the quotient $Z^{\prime}=Z \times{ }^{G} \mathrm{GL}_{n}$ is an algebraic space of finite presentation over $S$ and there is a natural isomorphism $X=\left[Z^{\prime} / \mathrm{GL}_{n}\right]$. Thus, we may assume that $G=\mathrm{GL}_{n}$. A map from $Y$ to $X$ is thus the same thing as an equivariant map from a $\mathrm{GL}_{n}$-torsor $T$ on $Y$ to $Z^{\prime}$, which is the same thing as a section of the bundle $Z_{Y}^{\prime} \times \mathrm{GL}_{n, Y} T \rightarrow Y$.

There is a universal $\mathrm{GL}_{n}$-torsor $T \rightarrow Y \times_{S} \operatorname{Hom}_{S}\left(Y, \mathrm{BGL}_{n}\right)$ which is representable by algebraic spaces. Forming the $Z^{\prime}$-bundle $Z_{\mathrm{Hom}_{S}\left(Y, \mathrm{BGL}_{n}\right)}^{\prime} \times \mathrm{GL}_{n} T \rightarrow$ $Y_{\operatorname{Hom}_{S}\left(Y, \mathrm{BGL}_{n}\right)}$, we can apply 2.3.3 to see that the natural map $\operatorname{Hom}_{S}(Y, X) \rightarrow$ $\operatorname{Hom}_{S}\left(Y, \mathrm{BGL}_{n}\right)$ is representable by algebraic spaces.

\section{Applications to BRANCHVARIETIES}

3.1. The proof of $\mathbf{1 . 2}$. Given the results of $\S 2.1$, the proof of 1.2 follows immediately from the following lemma concerning the locus of reduced fibers.

Lemma 3.1.1. Let $\pi: Y \rightarrow S$ be a proper flat morphism of finite presentation of Artin stacks. The locus over which the geometric fibers of $Y$ are reduced is an open substack of $S$.

Proof. Let $\rho: U \rightarrow Y$ be a smooth cover. By 12.2.1 of [6], the locus of points in $u \in U$ such that $U_{\pi(u)}$ is geometrically reduced at $u$ is open in $U$. Taking the image of this open subscheme by $\rho$ defines an open substack $V \subset Y$. Since $\pi$ is proper, $\pi(Y \backslash V) \subset S$ is closed. Since the property of being geometrically reduced is local in the smooth topology, it is easy to see that $S \backslash \pi(Y \backslash V)$ is the open set parametrizing geometrically reduced fibers of $Y$.

3.2. Existence of limits. We assume that $S=\operatorname{Spec} A$ is the spectrum of a discrete valuation ring. Let $Y / S$ be an arbitrary (not necessarily proper) Deligne-Mumford stack of finite type, and let $V:=U \times_{Y} U \rightrightarrows U \rightarrow Y$ be an étale presentation. Let $X_{\eta} \rightarrow Y_{\eta}$ be a finite morphism such that $X_{\eta}$ is geometrically reduced. Let $\mathscr{R}\left(X_{\eta}\right)$ be the integral closure of $\mathscr{O}_{Y}$ in $\mathscr{O}_{X_{\eta}}$, and let $\tilde{Y}=\operatorname{Spec} \mathscr{R} \rightarrow Y$.

Lemma 3.2.1. The formation of $\mathscr{R}$ commutes with étale base change $Y^{\prime} \rightarrow Y$.

Proof. This follows immediately from 6.14 .1 of [5]. Since this has a rather involved proof, we also offer a simpler alternative here. It is a tautology that the formation of $\mathscr{R}$ commutes with Zariski base change. Thus, it is easy to see that it suffices to prove this when $Y=\operatorname{Spec} B$ is a local scheme and $Y^{\prime}=\operatorname{Spec} B[x] /(f(x))\left[1 / f^{\prime}(x)\right]$ is a basic étale morphism (so $f(x)$ is monic of some degree $n$ ). Let $X=\operatorname{Spec} C$. Note that $B^{\prime}=\operatorname{Spec} B[x] /(f(x))$ is a finite free $B$-module. Calculations due to Tate (which may be found in $\S$ VII.1 of [13]) show that for any $y \in B^{\prime}, f^{\prime}(x) y=$ $\sum_{i=0}^{n-1} \operatorname{Tr}_{B^{\prime} / B}\left(b_{i} y\right) x^{i}$, with $b_{1}, \ldots, b_{n-1}$ certain elements of $B^{\prime}$. Let $C^{\prime}=B^{\prime} \otimes_{B} C$; since $B^{\prime} / B$ is étale away from $Z\left(f^{\prime}(x)\right), C^{\prime}$ is reduced after inverting $f^{\prime}(x)$. If $z \in C^{\prime}$ is any element integral over $B^{\prime}$, then $\operatorname{Tr}_{C^{\prime} / C} z$ is integral over $B$ (§5.1, Prop. 
$17 \mathrm{ff}$ of [2]). Applying the formula, we see that if $y \in C^{\prime}$ is integral over $B^{\prime}$, then $f^{\prime}(x) y \in B^{\prime}$ (as it is a polynomial in $x$ with coefficients in $B$ ). On the other hand, if $w$ is integral over $B^{\prime}\left[1 / f^{\prime}\right]$, then there is a multiple $\left(f^{\prime}\right)^{s} w$ which is integral over $B^{\prime}$. The result follows.

Lemma 3.2.2. If $X \rightarrow Y$ is a branchvariety, then the natural homomorphism $\operatorname{Spec} \mathscr{R} \rightarrow X$ is an isomorphism.

Proof. This is essentially Lemma 2.1 of [1]. (While they work with graded rings, their proof carries over verbatim.)

Lemma 3.2.3. Suppose $X \rightarrow Y$ and $X^{\prime} \rightarrow Y$ are branchvarieties. The natural restriction map

is an isomorphism.

$$
\rho: \operatorname{Isom}_{Y}\left(X, X^{\prime}\right) \rightarrow \operatorname{Isom}_{Y_{\eta}}\left(X_{\eta}, X_{\eta}^{\prime}\right)
$$

Proof. Given a generic isomorphism $\varphi: X_{\eta} \stackrel{\sim}{\rightarrow} X_{\eta}^{\prime}$, there is an induced isomorphism $\operatorname{Spec} \mathscr{R}\left(X_{\eta}\right) \rightarrow \operatorname{Spec} \mathscr{R}\left(X_{\eta}^{\prime}\right)$. By 3.2.2, this gives rise to an isomorphism $X \rightarrow X^{\prime}$. Thus, $\rho$ is surjective. On the other hand, $\mathscr{R}\left(X_{\eta}\right) \subset \iota_{*} \mathscr{O}_{X_{\eta}}$, where $\iota: X_{\eta} \rightarrow X$ is the inclusion of the generic fiber. This immediately implies that $\rho$ is injective.

Corollary 3.1. The stack of branchvarieties is separated with finite diagonal.

Proof. Lemma 3.2.3 is precisely the valuative criterion of properness for the diagonal (when $X=X^{\prime}$ ). Quasi-finiteness of the diagonal follows immediately from the fact that the automorphism group of a finite reduced algebra over a field is finite.

Proposition 3.2.4. There is a branchvariety $X \rightarrow Y$ extending $X_{\eta}$ if and only if there is a branchvariety $X \rightarrow U$ extending $X_{\eta} \times_{Y} U$.

Proof. The non-trivial part of the proposition is deducing the existence of $X$ from the existence of $X$. Consider the two pullbacks $X_{1}:=p_{1}^{*} X \rightarrow V$ and $X_{2}:=p_{2}^{*} X \rightarrow V$. Since $x_{\eta}$ descends to $Y$, there is an isomorphism $\left(X_{1}\right)_{\eta} \stackrel{\sim}{\rightarrow}\left(X_{2}\right)_{\eta}$ with trivial coboundary on $U \times_{Y} U \times_{Y} U$. By 3.2.3, this descent datum extends to a descent datum $X_{1} \stackrel{\sim}{\rightarrow} X_{2}$, yielding a finite $Y$-space $X \rightarrow Y$ and an isomorphism $X_{U} \stackrel{\sim}{\rightarrow} X$. Since $U \rightarrow Y$ is étale, the fact that $X \rightarrow U$ is a branchvariety immediately implies that $X \rightarrow Y$ is a branchvariety.

Lemma 3.2.5. Suppose $Y=\operatorname{Spec} R$ is an affine scheme of finite presentation over $S$. Given a generic branchvariety $X_{\eta} \rightarrow Y_{\eta}$, there is a totally ramified extension $A \subset A^{\prime}$ such that $X_{\eta} \otimes A^{\prime}$ extends to a branchvariety over all of $Y \otimes A^{\prime}$.

Proof. One way to prove this is to use Theorem 2.5 of [1]! Choose an affine embedding $Y \subset \mathbf{A}_{S}^{N}$, and let $\bar{Y} \subset \mathbf{P}_{S}^{N}$ be the projective closure of $Y$. Normalizing $\bar{Y}_{\eta}$ in $\mathscr{O}_{X_{\eta}}$ yields a generic branchvariety $\bar{X}_{\eta} \rightarrow \bar{Y}_{\eta}$ whose restriction to $Y_{\eta}$ is isomorphic to $X_{\eta}$. By the result of Alexeev and Knutson cited in the first sentence, there is a totally ramified extension $A \subset A^{\prime}$ and a branchvariety $\bar{X} \rightarrow \bar{Y} \otimes A^{\prime}$ extending $\bar{X}_{\eta} \otimes A^{\prime}$. Restricting $\bar{X}$ to $Y \otimes A^{\prime}$ yields the desired branchvariety $X \rightarrow Y \otimes A^{\prime}$.

Proof of 1.3. We may assume that $S=\operatorname{Spec} A$ is the spectrum of a discrete valuation ring. Since $Y$ is of finite presentation over $S$, it is quasi-compact, so we may choose an étale cover $U \rightarrow Y$ with $U$ affine. Applying 3.2.5 and 3.2.4 completes the proof. 
3.3. Applications to branchvarieties on polarized orbifolds. The most potentially interesting application of these results is to the study of branchvarieties on orbifolds with projective coarse moduli spaces. In this case, there will be enough numerical invariants to again produce proper stacks. More generally, there are two (related) situations under which one can prove that the components are quasicompact.

Theorem 3.3.1. Suppose $S$ is Noetherian. If there is a proper flat surjection $\mu: \Xi \rightarrow Y$ with $\Xi$ quasi-projective over $S$ then the connected components of $\mathfrak{B} r_{Y / S}$ are proper over $S$.

Proof. Note that the morphism sending $f: X \rightarrow Y$ to the coherent sheaf $f_{*} \mathscr{O}_{X}$ gives a finite-type morphism of stacks $\mathfrak{B} r_{Y / S} \rightarrow \mathrm{Coh}_{Y / S}$. Moreover, it sends a connected component into a connected component. Furthermore, the pullback $\mu^{*}$ gives a finite type homomorphism of algebraic stacks $\mathrm{Coh}_{Y / S} \rightarrow \mathrm{Coh}_{\Xi / S}$. It thus suffices to show that the connected components of the stack $\mathrm{Coh}_{\Xi / S}$ are of finite type over $S$. But this follows from Lemma 1.3 and Lemma 1.6 of [1]. Indeed, since $S$ is quasi-compact, there is an $S$-very ample invertible sheaf on $\Xi$ coming from a global immersion $\Xi \hookrightarrow \mathbf{P}_{S}^{N}$. Thus, it suffices to prove the result assuming $\Xi=\mathbf{P}_{S}^{N}$. On any connected component of $\mathrm{Coh}_{\mathbf{P}^{N} / S}$, the Hilbert polynomial and degree sequence are constant in fibers. By Kleiman's theorem, such sheaves have a bounded Castelnuovo-Mumford regularity, so they all appear as quotients of a fixed sheaf of the form $\mathscr{O}(-m)^{M}$ on $\mathbf{P}_{S}^{N}$. The rest follows by classical results of Grothendieck.

Remark 3.3.2. It is tempting to believe that if $Y$ is an algebraic space of finite presentation over $S$ and there is a proper flat map $\Xi \rightarrow Y$ with $\Xi$ a quasi-projective scheme, then $Y$ is in fact a quasi-projective scheme. When $S$ is the spectrum of a field, this is true. The proof proceeds by (locally on $S$ ) slicing $\Xi$ to produce a finite flat such morphism (as in [7]). Taking the norm of an ample invertible sheaf on the cover then produces an ample invertible sheaf on $Y$ (cf. $\S 6.6$ of [3]). When $S$ is larger than a point, we can at least see that if $Y$ admits a finite flat cover by a quasi-projective scheme then $Y$ is quasi-projective.

Theorem 3.3.3. Suppose $S$ is Noetherian and $Y$ is a tame quotient stack with quasi-projective coarse moduli space. The connected components of the stack $\mathfrak{B} r_{Y / S}$ are proper over $S$.

Proof. We can clearly assume that $S$ is connected. If $S$ is the spectrum of a field, then this follows from the fact [7] that $Y$ admits a finite flat cover by a projective scheme, combined with 3.3.1. In general we do not know that $Y$ admits a nice cover, but we can give an alternative proof.

Let $E$ be a generating sheaf for $Y$, as defined in [12]. Consider the morphism $\gamma_{E}: \operatorname{Coh}_{Y / S} \rightarrow \operatorname{Coh}_{\bar{Y} / S}$ defined by sending $F$ to $\pi_{*} \mathscr{H} o m(E, F)$ (that $\gamma_{E}(F)$ is flat over the base follows from the tameness of $Y$ ). By definition, there is a surjection $\pi^{*} \gamma_{E}(F) \otimes E \rightarrow F$. A connected component $\Gamma \subset \mathrm{Coh}_{Y / S}$ maps into a connected component of $\operatorname{Coh}_{\bar{Y} / S}$. By Kleiman's theorem, the connected components of $\mathrm{Coh}_{\bar{Y} / S}$ are proper. More precisely, there exist $m$ and $M$ such that for all $F \in \Gamma$ there is a surjection $\mathscr{O}(-m)^{M} \rightarrow \pi_{*} \mathscr{H} o m(E, F)$. Combining this with the definition of a generating sheaf, this yields a surjection $E(-m)^{M} \rightarrow F$. Moreover, since $\Gamma$ is connected, for any $T \rightarrow S$ and any lift $T \rightarrow \Gamma$, the function $P_{F_{t}}: K^{0}(Y) \rightarrow \mathbf{Z}$ 
sending a locally free sheaf $G$ on $Y$ to $\chi\left(\mathscr{H} o m\left(G_{t}, F_{t}\right)\right)$ is locally constant as $t$ varies (Lemma 4.3 of [12]). Thus, $\Gamma$ is the image of a closed subspace of the space $Q\left(E(-m)^{M}, P\right)$ of quotients of $E$ with Hilbert polynomial $P$. By 4.5 of [12], this space of quotients is quasi-projective over $S$, hence is quasi-compact. (The hypothesis in [12] that $S$ be an affine scheme is unnecessary for $Q(P)$ to be a quasi-compact algebraic space, but the reader uncomfortable with this bald assertion may choose to only regard this corollary as true under the additional hypothesis that $S$ is an affine scheme.) It follows that $\Gamma$ is quasi-compact, finishing the proof.

Of course, describing the connected components (or collections thereof) is quite a subtle task. The best one could hope for is to find numerical invariants which bound a collection of branchvarieties. As the proofs above show, once one has chosen a polarized cover or a generating sheaf and a polarization of the coarse moduli space, one can use the resulting numerical invariants to bound substacks of $\mathfrak{B} r_{Y / S}$.

If $S$ is the spectrum of a field and $Y$ is tame, smooth, and separated with quasiprojective coarse moduli space, then it is known [7] that $Y$ is a quotient stack (and that it admits a finite flat cover by a quasi-projective scheme). Thus, 3.3.1 and 3.3.3 both apply to show that the components of $\mathfrak{B} r_{Y / S}$ are proper. In this direction, it might be interesting to consider the scheme of branchvarieties of a weighted projective space. The corresponding Hilbert scheme plays an important role in recent work of Abramovich and Hassett on the moduli of stable varieties.

\section{REFERENCES}

[1] Valery Alexeev and Allen Knutson. Complete moduli spaces of branchvarieties, 2006. Preprint.

[2] Nicolas Bourbaki. Commutative algebra. Chapters 1-7. Elements of Mathematics (Berlin). Springer-Verlag, Berlin, 1998. Translated from the French, Reprint of the 1989 English translation.

[3] A. Grothendieck. Éléments de géométrie algébrique. II. Étude globale élémentaire de quelques classes de morphismes. Inst. Hautes Études Sci. Publ. Math., (8), 1961.

[4] A. Grothendieck. Éléments de géométrie algébrique. III. Étude cohomologique des faisceaux cohérents. II. Inst. Hautes Études Sci. Publ. Math., (17):223, 1963.

[5] A. Grothendieck. Éléments de géométrie algébrique. IV. Étude locale des schémas et des morphismes de schémas. II. Inst. Hautes Études Sci. Publ. Math., (24):231, 1965.

[6] A. Grothendieck. Éléments de géométrie algébrique. IV. Étude locale des schémas et des morphismes de schémas. III. Inst. Hautes Études Sci. Publ. Math., (28):255, 1966.

[7] Andrew Kresch and Angelo Vistoli. On covering of Deligne-Mumford stacks and surjectivity of the Brauer map. Bull. London Math. Soc., 36(2):188-192, 2004.

[8] Gérard Laumon and Laurent Moret-Bailly. Champs algébriques. Springer-Verlan, Berlin, 2000.

[9] Martin Olsson. Sheaves on Artin stacks. Preprint, 2005.

[10] Martin Olsson. Hom-stacks and restriction of scalars. To appear, Duke Math. J..

[11] Martin Olsson. On proper coverings of Artin stacks. Adv. Math, 198: 93-106, 2005.

[12] Martin Olsson and Jason Starr. Quot functors for Deligne-Mumford stacks. Comm. Algebra, 31(8):4069-4096, 2003. Special issue in honor of Steven L. Kleiman.

[13] Michel Raynaud. Anneaux locaux henséliens. Lecture Notes in Mathematics, Vol. 169. Springer-Verlag, Berlin, 1970.

[14] Jason Starr. Remarks on moduli spaces and Artin's axioms. Preprint, 2006. 